\title{
Metformin Drug Repurposing as A Potential Therapeutic Principle for Cancer Treatment
}

\author{
Dalia K Zaafar ${ }^{1}$ and Soha 0 Hassanin*2 \\ ${ }^{1}$ Department of Pharmacology, Faculty of pharmacy, Egypt \\ ${ }^{2}$ Department of Biochemistry, Faculty of pharmacy, Egypt \\ *Corresponding author: Soha O Hassanin, Department of Biochemistry, Faculty of pharmacy, Egypt
}

\begin{tabular}{|c|c|}
\hline ARTICLE INFO & ABSTRACT \\
\hline Received: 㓞 December 16, 2020 & $\begin{array}{l}\text { Citation: Dalia K Zaafar, Soha } 0 \text { Hassanin. Metformin Drug Repurposing as A Potential } \\
\text { Therapeutic Principle for Cancer Treatment. Biomed J Sci \& Tech Res 32(5)-2020. BJSTR. }\end{array}$ \\
\hline Published: December 23, 2020 & MS.ID.005307. \\
\hline
\end{tabular}

\section{Introduction}

Cancer is a major health concern worldwide and is predicted to raise the global burden of cancer in the coming years [1]. Growth in the population, improved life expectancy and economic progress are main causes of a continuing rise in the burden of cancer. In 2025, more than 20 million individuals will be diagnosed with cancer, according to global statistics [1]. Malignancies such as colorectal, prostate and breast cancer, which are often incurable with existing therapies in advanced stages, would be a major contributor to this rise. Therefore, tackling these current and future challenges requires more effective drugs for cancer.

Although massive investments have been pushed into drug production, there is only a small success. Since the 1990s, the total number of FDA approvals per year has decreased. The average length of time between initial studies and the completed regulatory review ranges from 11.4 to 13.5 years. Generally, figures of capitalized costs differ, ranging from 2-3 billion dollar per drug till hitting the market [2]. The need for more effective anti-cancer drugs has ignited a growing interest for drug repurposing, which is a concept for using drugs already approved originally for other uses to treat cancer by either inducing apoptosis or suppressing proliferation. As a result, comprehensive data are available, further decreasing the need for further research to investigate the pharmacokinetic properties and toxicity. It is likely that the safety profile resembles that of the initial indication $[3,4]$. To maintain proliferation, rapamycin, indomethacin and prazosin are proposed, while artemisinin and chloroquine induce apoptosis and metformin can regulate cell metabolism [5]. The anticipated function and mechanism of metformin as a possible treatment for cancer will be addressed in this mini review.

\section{Metformin}

Several studies have confirmed that there has been a lower incidence of colorectal, hepatocellular, pancreatic, stomach, liver, oesophagus and lung cancer in users of metformin. A multicentral metformin cohort study showed that metformin users had a reduced risk of developing cancer, even after various demographic features, metabolic parameters and diabetic complications had been adjusted [6]. The use of metformin has been associated with a favorable response to therapy and improved survival in cancers such as hepatocellular carcinoma, colorectal, prostate, HER2+ breast cancer, ovarian, pancreatic, esophageal and rectal cancer in diabetes patients with proven cancer. This is mediated by mitochondrial respiration interference, reducing citric acid cycle activity and overall ATP production, but cancer cells can counteract this effect by increasing ATP production through elevated aerobic glycolysis in a compensatory manner. Thus, the anti-neoplastic activity of metformin depends on the ability of cancer cells to enable the production of glycolysis-mediated ATP and the availability of tissue glucose.

In addition, cancer cells with mutations in the genes of the respiratory complex I and therefore unable to control oxidative phosphorylation are more susceptible to metformin. By phosphorylation, metformin also activates AMP-activated protein kinase (AMPK) [7]. There are many downstream effects of AMPK, 
among them anabolic pathway inhibition and decreased protein synthesis. With mTOR down-regulation, AMPK is associated. Indirectly, metformin also activates AMPK via the liver kinase B11 tumor suppressor (LKB1). Mechanistically, metformin mTOR inhibition tends to occur at the translation level, and metformin therapy contributes to mRNA translation suppression in general, but also specifically in a series of cancer-promoting genes. In breast cancer cells, metformin-treated growth inhibition is mediated by activation of AMPK and altered translation of mRNA by inhibition of translational proteins such as ribosomal S6 kinase and eIF4Ebinding protein 1 . In a study of colon cancer in mice in which apoptosis and antiproliferation were induced by metformin, a similar mechanism was documented (Zaafar, Zaitone, and Moustafa 2014). In addition, inhibition of factors 4E-BP1 and EF2, also essential for translation, is regulated by metformin activation of AMPK downstream. AMPK also inhibits the unfolded protein response (UPR) in leukemic cells, which is usually activated to remove improperly folded proteins in the endoplasmic reticulum. Thus, treatment with metformin can cause an accumulation of ER stress via unfolded proteins that are not removed due to UPR defects, resulting in apoptosis. Metformin's predicted anti-cancer effect was summarized in the Figure 1.

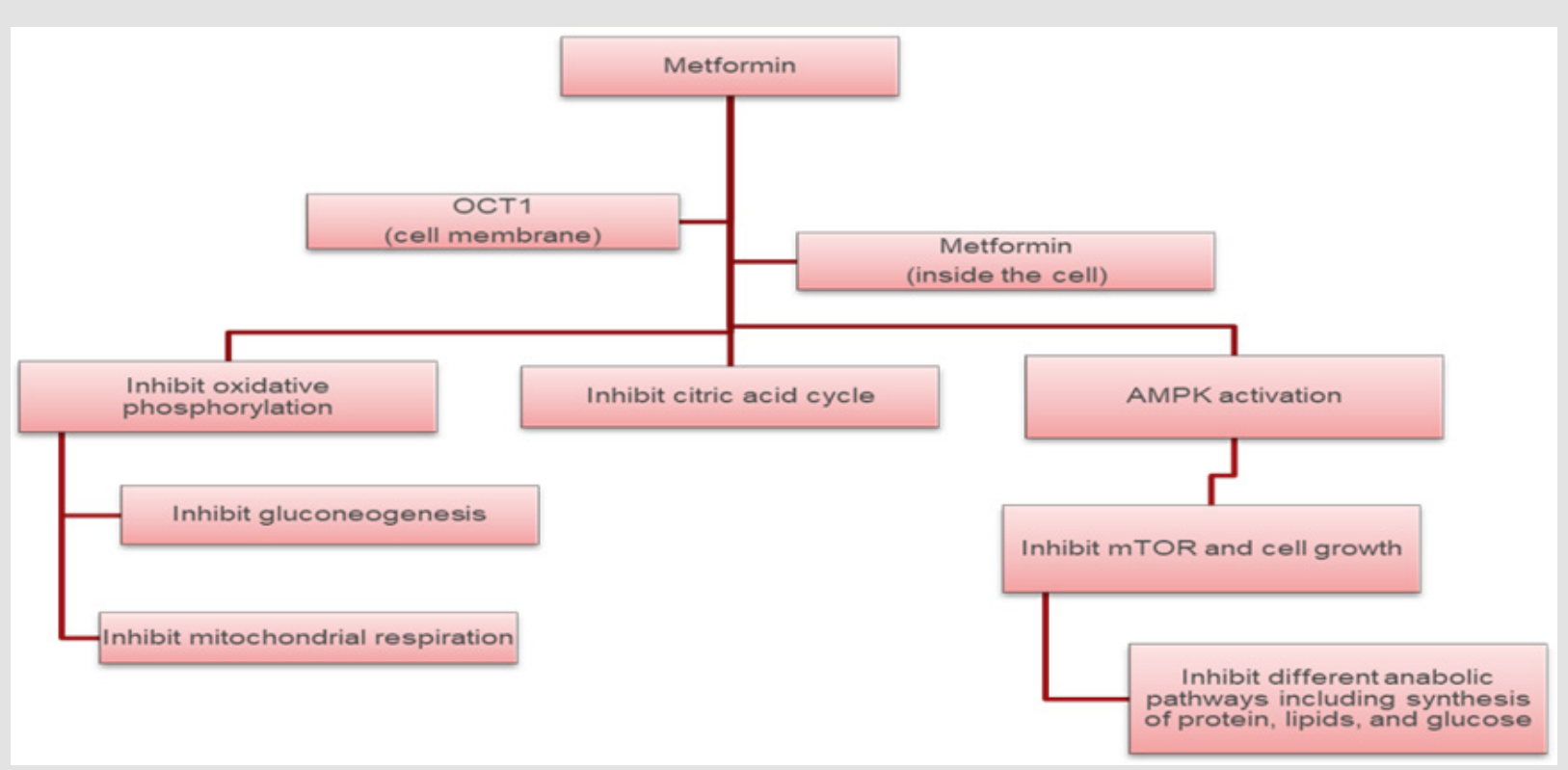

Figure 1: Shows the different metformin mechanisms in treating cancers. Oct1: organic cation transporter 1, AMPK: AMP activated protein kinase, mTOR: Mechanistic target of rapamycin.

\section{References}

1. F Bray, J Ferlay, I Soerjomataram, RL Siegel, LA Torre, et al. (2018) Global cancer statistics 2018: GLOBOCAN estimates of incidence and mortality worldwide for 36 cancers in 185 countries, CA. Cancer J. Clin 68(6): 394424.

2. M Su, Q Zhang, X Bai, C Wu (2017) Availability, cost, and prescription patterns of antihypertensive medications in primary health care in China: A nationwide cross-sectional survey. Lancet 390(10112): 25592568.

3. S Pushpakom, F Iorio, P Eyers, K Jane, S Hopper, et al. (2019) Drug repurposing: progress, challenges and recommendations, Nature Reviews 18(2019): 41-58.

ISSN: 2574-1241

DOI: $10.26717 / B J S T R .2020 .32 .005307$

Soha 0 Hassanin. Biomed J Sci \& Tech Res

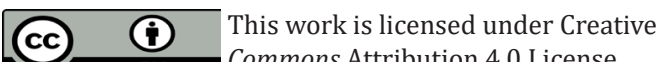

Submission Link: https://biomedres.us/submit-manuscript.php
4. Pan P (2017) Scientific advice-is drug repurposing missing a trick? Nature Reviews Clinical Oncology 14(8).

5. Z Zhang, Li Z, Na X, EC Nice, Tao Z, et al. (2020) Overcoming cancer therapeutic bottleneck by drug repurposing, Signal Transduction and Targeted Therapy 5(1)

6. HJ Kim, SJ Lee, KH Chun, JY Jeon (2018) Metformin reduces the risk of cancer in patients with type 2 diabetes, Medicine (Baltimore) 97(8): e0036.

7. B Viollet, B Guigas, N Sanz Garcia, J Leclerc, M Foretz, et al. (2012) Cellular and molecular mechanisms of metformin: An overview, Clinical Science122(6): 253-270.

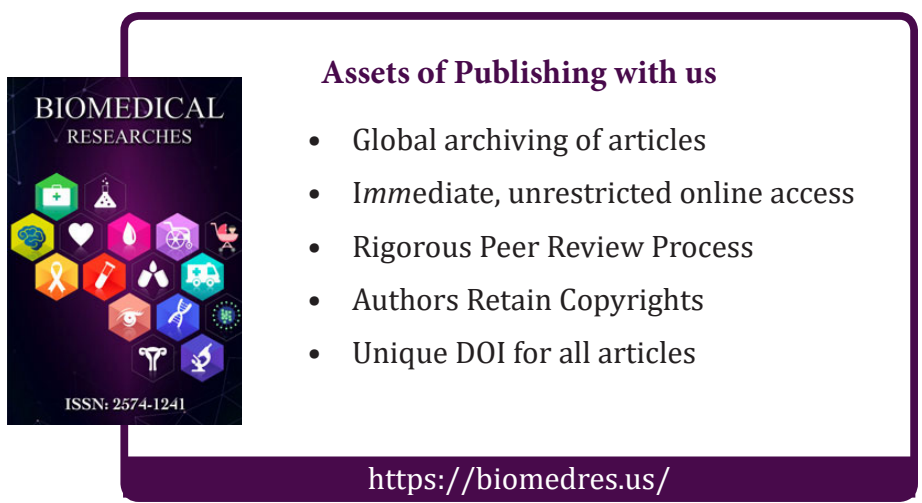

